\title{
Principles underlying efficient exciton transport unveiled by information-geometric analysis
}

\author{
Scott Davidson $\odot,{ }^{1, *}$ Felix A. Pollock, ${ }^{2, \dagger}$ and Erik Gauger ${ }^{1, \dagger}$ \\ ${ }^{1}$ SUPA, Institute of Photonics and Quantum Sciences, Heriot-Watt University, Edinburgh EH14 4AS, United Kingdom \\ ${ }^{2}$ School of Physics and Astronomy, Monash University, Clayton, Victoria 3800, Australia
}

(Received 8 May 2020; revised 31 May 2021; accepted 8 June 2021; published 1 July 2021)

\begin{abstract}
Adapting techniques from the field of information geometry, we show that open quantum system models of Frenkel exciton transport, a prevalent process in photosynthetic networks, belong to a class of mathematical models known as "sloppy." Performing a Fisher-information-based multiparameter sensitivity analysis to investigate the full dynamical evolution of the system and reveal this sloppiness, we establish which features of a transport network lie at the heart of efficient performance. We find that fine tuning the excitation energies in the network is generally far more important than optimizing the network geometry and that these conclusions hold for different measures of efficiency and when model parameters are subject to disorder within parameter regimes typical of molecular complexes involved in photosynthesis. Our approach and insights are equally applicable to other physical implementations of quantum transport.
\end{abstract}

DOI: 10.1103/PhysRevResearch.3.L032001

Introduction. Energy transport processes are prevalent across science and engineering, from biochemical reactions supporting life $[1,2]$ to nowadays ubiquitous electronic devices $[3,4]$. Typically, the goal of this process is to guide an excitation from a "source" to a "sink" as quickly and reliably as possible; however, a trade-off between quick versus robust transport often arises. Recently, there has been increasing interest in nanoscale energy transport due to the impressive efficiency of photosynthetic processes [5], potential enhancements in solar cell efficiency [6,7], and for other nanotechnology applications [8]. Any practical technology will need to overcome many uncontrollable degrees of freedom, arising from vibrations, the electromagnetic environment, and various quantum mechanical effects. Open quantum systems theory is thus an ideal basis for modeling these transport processes [9-12].

Photosynthetic exciton transport networks have inspired much theoretical work investigating the interplay between unitary time evolution and environment-induced decoherence [13-18]. Many previous studies have focused on the relationship between network geometry (via position-dependent dipole couplings) and transport efficiency: For example, for purely coherent transport, Ref. [19] reports statistical correlations between robust transport and geometries with a "backbone-pair" structure. Similarly, Refs. [20-22] compare the effects of coherent transport, phenomenological site-basis

\footnotetext{
*sd109@hw.ac.uk

$\dagger$ felix.pollock@monash.edu

*e.gauger@hw.ac.uk
}

Published by the American Physical Society under the terms of the Creative Commons Attribution 4.0 International license. Further distribution of this work must maintain attribution to the author(s) and the published article's title, journal citation, and DOI. pure dephasing, and network geometry. In the above cases the on-site energies were degenerate and assumed to be unimportant. By contrast, nondegenerate site energies were used for investigating statistical correlations between dynamics and network design [23,24], and studying optimal trapping processes [25]. In these studies, pure dephasing serves to overcome (Anderson) localization effects [26]. Moving beyond pure dephasing, Ref. [27] investigates geometrical effects with a more realistic environment model, whereas Refs. [28,29] incorporate vibronic effects. Finally, Ref. [30] analyzes transport efficiency and robustness in different vibrational parameter regimes with fixed, nondegenerate on-site energies.

In this Letter, we use information-geometric tools to show that, with realistic noise models, on-site energy tuning is typically far more important to transport performance than spatial configuration. While we focus on (Frenkel) exciton transport through a small network of sites, in a regime appropriate to the early stages of photosynthesis, our results are equally relevant to the design of organic photovoltaics, molecular charge transfer systems, and quantum walks more generally [31-33]. Constructing the Fisher information matrix (FIM) for our transport model, we show that its parameter space possesses a surprising structure which is common to mathematical models across scientific disciplines from biology to machine learning. These models have been termed "sloppy" since they meaningfully depend on only a few key parameter combinations [34]. We proceed by exploiting this property to analyze which physical parameters must be controlled most carefully in order to realize efficient quantum transport. Finally, we investigate how variations in both the transport model and employed efficiency measure affect this parameter sensitivity. Going beyond phenomenologically informed previous works, we elucidate how details of the vibrational environment determine whether the energetic landscape or network geometry most significantly influence the system's 
transport properties, confirming that in molecular networks the former typically dominates.

Transport model. We consider a network comprising $N$ sites, each modeled as a two-level system, and assume there is never more than a single excitation present. The unitary dynamics are governed by the Hamiltonian

$$
\hat{\mathcal{H}}_{s}=\sum_{i=1}^{N} E_{i}|i\rangle\langle i|+\sum_{i \neq j} V_{i j}(|i\rangle\langle j|+| j\rangle\langle i|),
$$

with intersite coupling strength $V_{i j}$ between site-basis states $|i\rangle$ and $|j\rangle$, and on-site excitation energy $E_{i}$ relative to the ground state $|g\rangle$. For simplicity, we present the case $N=4$, however, as shown in the Supplemental Material (SM) [35], our conclusions hold for other small networks. The dipolar hopping terms in Eq. (1) are given by $V_{i j}\left(\vec{r}_{i}, \vec{r}_{j}\right)=D /\left|\vec{r}_{i}-\vec{r}_{j}\right|^{3}$, where $\vec{r}_{i}$ denotes the real-space coordinates of site $|i\rangle$ and $D$ is a coupling constant, assumed equal for all sites.

Additionally, we account for a finite-temperature phonon environment, which couples to our transport network through site-dependent displacements with interaction Hamiltonian $\hat{\mathcal{H}}_{I, \text { phonon }}=\sum_{i}^{N}|i\rangle\langle i| \otimes \sum_{k} g_{k}\left(\hat{c}_{i, k}+\hat{c}_{i, k}^{\dagger}\right)$, where $\hat{c}_{i, k}^{(\dagger)}$ is the annihilation (creation) operator of local phonon mode $k$ and $g_{k}$ is the associated coupling constant. For computational convenience, we assume weak to moderate system-environment couplings that are adequately described with a Bloch-Redfield master equation [9]. This vibrational environment leads to phonon-mediated eigenstate transitions with rates determined by the phonon spectral density $J(\omega)$ [36]. form

We focus on a structured, singly peaked spectrum of the

$$
J_{1}(\omega)=\gamma_{\mathrm{ph}}\left|\frac{\omega}{\omega_{c}}\right|^{3} e^{-\left(\frac{\omega}{\omega_{c}}\right)^{2}}\left[n_{\mathrm{BE}}\left(\omega, T_{\mathrm{ph}}\right)+\Theta(\omega)\right],
$$

where $\gamma_{\mathrm{ph}}$ controls the overall system-phonon coupling, the Bose-Einstein distribution $n_{\mathrm{BE}}$ describes a phonon environment at temperature $T_{\mathrm{ph}}$, the Heaviside step function $\Theta(\omega)$ reflects the prevalence of phonon emission over absorption at finite temperature, and $\omega_{c}$ is a high-frequency cutoff. This generic form captures the typical situation of a vanishing $J(\omega)$ in both low- and high-frequency limits. Additionally, we consider two other functional forms, namely $J_{2}(\omega)=$ $\gamma_{\mathrm{ph}} \Theta(\omega)$ and $J_{3}(\omega)=\gamma_{\mathrm{ph}}$, to investigate which features of $J_{1}$ are critical to our findings. Physically, $J_{3}$ represents a flat, infinite-temperature spectrum which, while unrealistic in practice, leads to Markovian pure dephasing (used in many previous exciton transport studies [20-23]) within our nonsecular Bloch-Redfield treatment, thus allowing for a comparison with the existing literature. Spectrum $J_{2}$ describes a flat, zerotemperature environment and acts as a bridge between $J_{1}$ and $J_{3}$ by retaining the thermodynamically consistent asymmetry between phonon absorption and emission while removing all other structure.

Finally, we account for two other physical processes of relevance to energy transport phenomena. After successful transport, the Lindblad operator $\hat{L}_{t}=\gamma_{\text {trap }}^{1 / 2}|\operatorname{trap}\rangle\langle\operatorname{sink}|$ incoherently extracts an excitation from the designated network sink to an orthogonal trap state [Fig. 1(a)]. We also include spontaneous decay via $\hat{L}_{d, i}=\tau_{i}^{-1 / 2}|g\rangle\langle i|$, which destroys an excitation at site $|i\rangle$.

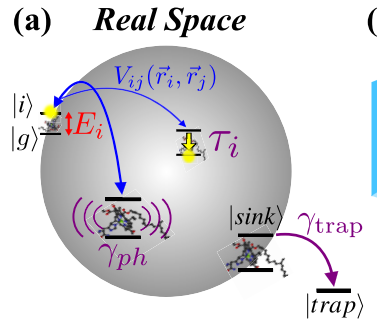

(b) Prediction Space

FIG. 1. (a) Schematic depiction of the nanoscale transport systems considered here. Our models account for coherent transport $\left(V_{i j}\right)$, vibrational coupling $\left(\gamma_{\mathrm{ph}}\right)$, spontaneous decay $\left(\tau_{i}\right)$, and energy extraction $\left(\gamma_{\text {trap }}\right)$. (b) Cartoon depiction of the parameter space manifold revealed by our information-geometric analysis and the associated log-linear ("sloppy") Fisher information matrix (FIM) eigenvalue spectrum which suggests that on-site excitation energies are the dominant parameters in determining transport performance.

Overall, the transport dynamics of our model is governed by the Bloch-Redfield equation

$$
\frac{\partial \rho_{s}}{\partial t}=-i\left[\hat{\mathcal{H}}_{s}, \rho_{s}\right]+\hat{\mathcal{R}} \rho_{s}+\hat{\mathcal{L}}\left[\hat{L}_{t}\right] \rho_{s}+\sum_{i=1}^{N} \hat{\mathcal{L}}\left[\hat{L}_{d, i}\right] \rho_{s},
$$

where subscript $s$ denotes system quantities, $\hat{\mathcal{R}}$ is our phonon interaction Bloch-Redfield tensor, and $\hat{\mathcal{L}}[\bullet] \rho$ is the usual Lindblad dissipator [9].

As a means to parametrize our transport networks in terms of physically relevant quantities (see Fig. 1), we use the following set of model parameters throughout this Letter: on-site excitation energies $E_{i}$; exciton lifetimes $\tau_{i}$; real-space polar coordinates $\vec{r}_{1 i}=\left(r_{1 i}, \phi_{1 i}, \theta_{1 i}\right)$ with respect to site $|1\rangle$; system to trap extraction rate $\gamma_{\text {trap }}$; system-environment vibrational coupling $\gamma_{\mathrm{ph}}$; and phonon bath temperature $T_{\mathrm{ph}}$. We denote the vector containing all of these input parameters as $\vec{\eta}=\left\{\eta_{\mu}\right\}$. Additional parametrization details and parameter values for our results are provided in the SM [35].

Given sufficient computational resources, the ensuing analysis could instead utilize other, more accurate numerical methods for simulating open quantum dynamics [37] such as polaron-transform-based master equations [38-40], hierarchical equations of motion [41,42], time-dependent density matrix renormalization group approaches [43,44], or path-integral-based approaches [45-47]. More broadly, our approach is equally applicable to far more complex models of physical systems than the simple tight-binding models considered here.

Fisher information matrix. Our primary focus is the nonequilibrium transport properties of a single energy quantum that is generated, transported, and extracted before another excitation enters the system, reflecting excitation events that are rare or transient. However, as shown later, our general conclusions also hold in steady-state scenarios. For now, to investigate transport efficiency over the full time evolution of the system, we consider the probability distribution $f_{\text {arr }}(t, \vec{\eta})=\left(P_{\max }\right)^{-1} \frac{\partial}{\partial t}\langle\operatorname{trap}|\rho(t, \vec{\eta})| \operatorname{trap}\rangle$, where $P_{\max }$ is the final trap population. This "arrival time" distribution is the likelihood of the excitation arriving at the trap at time $t$, normalized by the total probability of successful transport. Using this, measures of transport efficiency such as the mean 

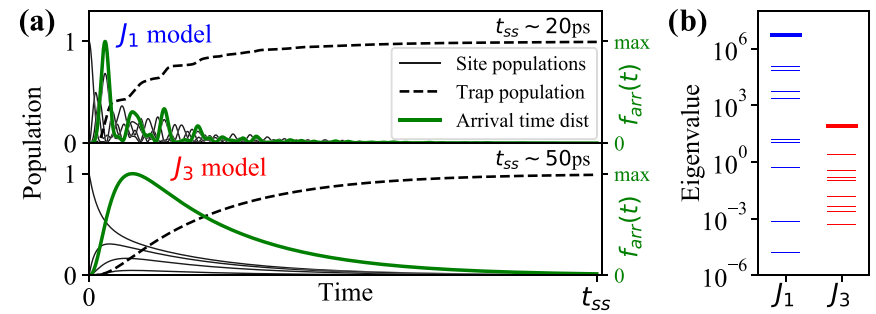

FIG. 2. Comparison between transport models with $J_{1}$ (phononmediated eigenstates transitions) and $J_{3}$ (site-basis pure dephasing) vibrational environments with all other parameters identical. (a) Population dynamics up to respective steady-state times $\left(t_{\mathrm{ss}}\right)$ for each system site (black lines) and trap state (dashed line) with associated arrival time distribution ( $f_{\text {arr }}$, green) on the right-hand axis. (b) Sloppiness of FIM eigenvalue spectra.

and variance of the arrival time can be computed. Our goal is to determine which, and how many, of our model's physical input parameters $(\vec{\eta})$ contribute most to determining these properties. We explore the parameter space of our models with the Fisher information matrix (FIM) [48] which, for a distribution $f(t, \vec{\eta})$, is defined as

$$
g_{\mu \nu}(\vec{\eta})=E\left[\left(\frac{\partial}{\partial \eta_{\mu}} \ln f(t, \vec{\eta})\right)\left(\frac{\partial}{\partial \eta_{\nu}} \ln f(t, \vec{\eta})\right) \mid t\right],
$$

where $E[\cdots \mid t]$ denotes the expectation value with respect to time. The FIM (4) is a metric tensor for the parameter space manifold (i.e., subspace of possible model outputs) embedded within the full "prediction space" of possible distributions [34]. Following Ref. [49], an intuitive geometric structure emerges from the FIM, in which the manifold possesses a hierarchy of widths (i.e., geodesic lengths) corresponding to successive, exponentially less important linear combinations of model parameters (see Fig. 1). Similar sloppy geometric structures are present in many unrelated mathematical models, from a variety of scientific disciplines [50-52]. Formally, the sloppiness of a particular model is determined by the manifold's widths along each of the FIM eigenvector directions. However, following Transtrum et al. [34,53], the FIM eigenvalues may be used as the key indicator of model sloppiness. Throughout this Letter we construct the FIM using dimensionless (log) parameters to allow for a fair comparison between parameters with different physical dimensions (see SM [35] for details).

Results and discussion. We begin by investigating how different vibrational environment descriptions affect the robustness of transport properties, as quantified by the sloppiness of the model. Specifically, we compare spectra $J_{1}$ and $J_{3}$ for a transport model with linear chain geometry and degenerate on-site energies, finding that this leads to two contrasting parameter space manifolds, where the transport properties are several orders of magnitude more sensitive to parameter perturbations for the first model $\left(J_{1}\right)$ than the second $\left(J_{3}\right)$, as seen by the relative difference in FIM eigenvalues. In the SM [35] we show that this contrast holds over a wide range of $\gamma_{\mathrm{ph}}$ values. The population dynamics in Fig. 2(a) show that only the $J_{1}$ model supports coherent (site-basis) oscillations on timescales relevant to the transport process. In this case, the resulting interference effects contribute to a dynamics that

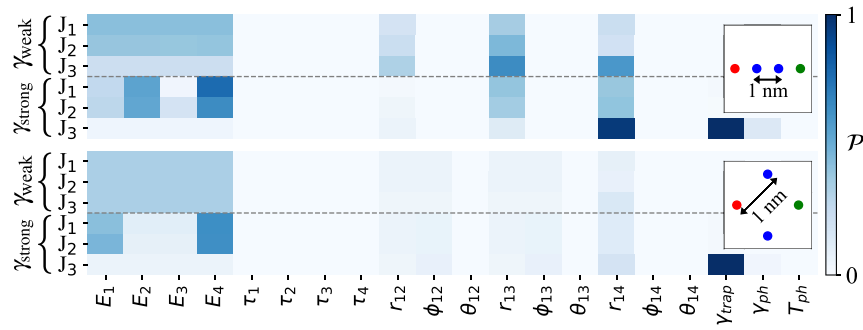

FIG. 3. Relative importance $\mathcal{P}\left(\eta_{\mu}\right)$ of each model parameter $\eta_{\mu}$ for simple $1 \mathrm{D}$ and $2 \mathrm{D}$ transport geometries. Rows correspond to different functional forms of the phonon spectrum [Eq. (2)] for both weak $\left(\gamma_{\text {weak }}=10^{-3} \mathrm{eV}\right)$ and stronger $\left(\gamma_{\text {strong }}=10^{-1} \mathrm{eV}\right)$ phonon coupling $\left(\gamma_{\mathrm{ph}}\right)$. Insets depict network geometries with red (green) dots representing the source (sink) site.

is far more sensitive to the details of the model. However, both models are inherently sloppy; the sensitivity to small parameter changes is concentrated in a few linearly independent perturbations, which we will now see primarily involve the on-site excitation energies.

We proceed by analyzing the FIM eigenvectors to perform a sensitivity analysis across different parameter regimes. This tells us which physical model parameters have the largest influence on the dynamical evolution of the system. The linear combination of bare model parameters which forms each eigenvector, paired with the magnitude of its eigenvalue, establishes the local (in parameter space) hierarchy of parameter sensitivities. In the SM [35] we show that, due to the inherent complexity of these transport networks, and the competing influences of various parameters, the parameter space lacks clear global patterns in these important linear combinations. Therefore, we instead look for bare parameters which consistently dominate the eigenvector(s) with the largest eigenvalue(s) (i.e., we identify a lower-dimensional parameter subspace which influences transport behavior significantly). To quantify relative parameter importance we use $\mathcal{P}\left(\eta_{\mu}\right)=\sum_{i} \lambda_{i}\left|\hat{e}_{i} \cdot \hat{\eta}_{\mu}\right|$ and normalize such that $\sum_{\mu} \mathcal{P}\left(\eta_{\mu}\right)=$ 1 , where $\hat{e}_{i}\left(\lambda_{i}\right)$ is the $i$ th eigenvector (eigenvalue) of the FIM, and $\hat{\eta}_{\mu}$ is the parameter space basis vector for model parameter $\eta_{\mu}$. We use absolute values since we are concerned with the magnitude of the sensitivity. This method establishes the relative parameter importance over the full dynamical transport process instead of focusing on the sensitivity of a single scalar quantity.

The quantity $\mathcal{P}\left(\eta_{\mu}\right)$ is shown in Fig. 3 for each physical input parameter $(\vec{\eta})$ in simple one- and two-dimensional (1D and 2D) geometries with different phonon environments. For weak system-phonon coupling, the parameters which control the system's unitary dynamics (i.e., positions and on-site energies) are significantly more important. Furthermore, we see a close similarity between $J_{1}$ and $J_{2}$ but, for pure-dephasinglike noise $\left(J_{3}\right)$, there are qualitative differences in parameter importance. This suggests that the presence of an asymmetry in phonon absorption and emission rates, derived from the thermodynamic consistency of models with spectra $J_{1}$ and $J_{2}$, is a key feature in determining which parameters are most important in exciton transport. Physically, a phonon rate asymmetry leads to lower-energy eigenstates becoming 

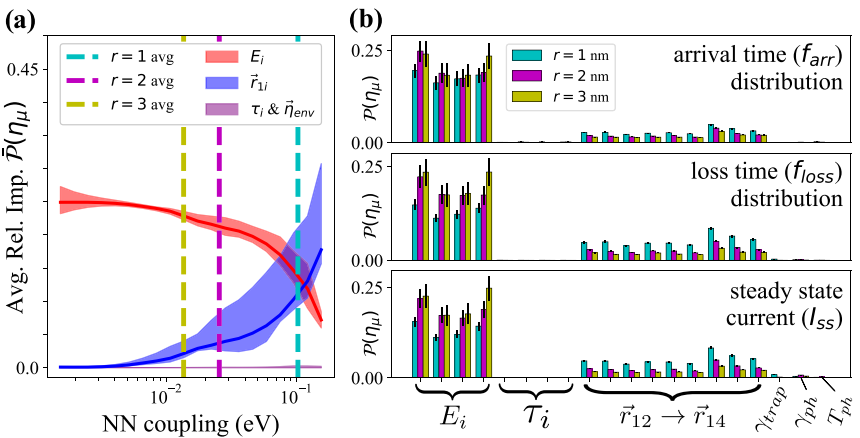

FIG. 4. (a) Average (solid lines) and range (shaded area) of relative importance for different parameter groups upon varying site spacing in a linear chain geometry (i.e., all pairwise couplings change but nearest-neighbor couplings are shown for simplicity). (b) Average parameter importance (main bar) and associated variance (error bar) for different transport efficiency measures using randomly generated four-site networks (1000 per $r$ value). Geometries were constrained within a sphere of radius $r$ with $10 \%$ disorder applied to all other model parameters (see main text and SM [35]). Dashed vertical lines in (a) denote average nearest-neighbor coupling across all networks for each of the three different sphere radii in (b).

increasingly populated as the evolution progresses. Therefore, the precise properties of these eigenstates will become more important. Without this asymmetry, and in the absence of an extraction process, the system would tend towards a maximally mixed state with equal population on all sites. In that case, the precise details of the system Hamiltonian and its resulting eigenstates will be less important, and site positions and excitation energies will have less influence on the system's dynamics. Finally, we note that stronger phonon coupling tends to exaggerate the above-discussed effects: For $J_{1}, J_{2}$, the lower-energy states are populated more quickly and so the details of these states, and the different on-site energies and positions which determine them, are more important. Similarly, for strong pure dephasing, the maximally mixed state is reached more quickly, therefore, the most important considerations are simply the extraction rate $\left(\gamma_{\text {trap }}\right)$ and the proximity of the sink site to all others.

For the remainder of this Letter, we focus on the $J_{1}$ phonon environment and turn to look more closely at the Hamiltonian parameters. We find that there is a connection between coherent coupling strength and the relative importance of network geometry versus on-site energies. Figure 4(a) compares these two parameter groups for a degenerate linear chain, showing that network geometry becomes more important as intersite coupling increases. However, on-site energies are dominant up to nearest-neighbor hopping around $100 \mathrm{meV}$. This can be explained as follows: As coherent couplings increase, eigenenergy splittings also increase. For structured spectral densities, such as $J_{1}$, this entails larger differences between rates of the various phonon-mediated eigenstate transitions and therefore different transport performance. In the SM [35] we show that this energy-position importance crossover disappears for pure-dephasing models. The shaded regions in Fig. 4(a) are bounded by the maximal/minimal values of $\mathcal{P}\left(\eta_{\mu}\right)$ when restricting $\vec{\eta}$ to encompass only the corresponding parameter group.
Figure 4(b) corroborates the dominant importance of onsite energies across a large region of parameter space. Its panels show the average relative parameter importance for randomly generated network geometries inside a sphere of radius $r$ with source and sink sites fixed at opposite poles and intermediate sites randomly positioned [see Fig. 1(a)], as in Refs. [20-22]. Consequently, the average coherent coupling strength is inversely related to $r$. Random disorder is also applied to all other parameters (see SM [35] for procedural details). In all cases, we focus on networks exhibiting relatively efficient transport by restricting to randomly generated networks whose time evolution lasts less than $1 \mathrm{~ns}$. The top panel shows results for the arrival time distribution $f_{\text {arr }}(t, \vec{\eta})$. The middle panel uses an alternative indicator of successful transport, namely the loss time distribution $f_{\text {loss }}(t, \vec{\eta})=$ $\left(P_{\text {loss }}\right)^{-1} \frac{\partial}{\partial t}\langle g|\rho(t, \vec{\eta})| g\rangle$ which describes the probability of exciton recombination at time $t$, normalized by the overall recombination probability $P_{\text {loss }}$. Finally, with previous studies arguing that, for photosynthetic systems under incoherent illumination, only steady-state transport properties are relevant $[15,54]$, we also consider the steady-state current into the trap, $I_{\mathrm{ss}}=\gamma_{\text {trap }}\left\langle\operatorname{sink}\left|\rho_{\mathrm{ss}}(\vec{\eta})\right| \operatorname{sink}\right\rangle$, which necessitates adding an injection term $\hat{\mathcal{L}}\left[\sqrt{\gamma_{\text {inj }}} \hat{\sigma}_{1}^{+}\right] \rho_{s}$ to Eq. (3) for repopulating the source site. Here, we simply look at the magnitudes of the partial derivatives $\left(\left|\partial_{\mu} I_{\mathrm{ss}}\right|\right)$ for parameter sensitivities. However, our nontrivial information-geometric approach could be adapted to steady-state scenarios by choosing $f(\vec{\eta})$ in Eq. (4) to be the full counting distribution of transported excitons [55].

As shown in Fig. 4(b), upon moving away from the idealized degenerate linear chain towards disordered model parameters, the importance of tuning the on-site energies dominates that of network geometry, regardless of the adopted efficiency measure. By comparing with the colored vertical lines in Fig. 4(a), we see that the transition from importance of on-site energies to network geometries at stronger couplings-as seen in Fig. 4(a)—no longer occurs, even for $r=1 \mathrm{~nm}$, where average nearest-neighbor couplings approach $100 \mathrm{meV}$. While all three efficiency measures show positions becoming slightly more important with decreasing sphere radii, we nonetheless conclude that, on average, tuning the on-site energies remains far more important for achieving efficient transport.

Finally, since the magnitude of intersite couplings in a typical photosynthetic system is $\lesssim 10 \mathrm{meV}$, our results suggest that on-site energy tuning, rather than molecular geometry, is primarily responsible for the impressive efficiency of natural photosynthetic processes. See SM [35] for an extended discussion of experimental platforms to which our results apply.

Conclusion. We have demonstrated that open quantum system models of exciton transport are sloppy, in common with many other multiparameter models from various scientific domains. For the generic transport models studied here, we found the extent of the sloppiness to depend on the nature of the phonon environment and, in particular, the coupling strength and finite-temperature asymmetry in phonon rates. Making use of the intrinsic hierarchy of parameter sensitivities implied by the sloppiness of our model, we performed an information-geometric multiparameter sensitivity analysis to understand which physical features of the system most affect 
its transport performance. We anticipate that the methodology introduced here could become an important asset for analyzing a host of other settings involving complex quantum dynamics, complementing other approaches that aim to capture its key features [56-58]. Crucially, it also opens the door to the application of other information-geometric analyses $[51,53,59]$.

Our results underline the importance of a tailored on-site energy landscape for achieving efficient quantum transport [24] and demonstrate considerable robustness to parameter disorder. Intuitively, this parameter importance reflects the fact that on-site energies have a direct influence on both the unitary and nonunitary dynamics through their effects on the eigenenergies and eigenstates of the system, therefore governing coherent as well as dissipative processes. Our findings suggest the future exploration of nontrivial energy landscapes, such as those recently found in Ref. [60], could lead to significant improvements in our understanding and implementation of efficient quantum transport processes.

Acknowledgments. S.D. was supported by the EPSRC Grant No. EP/L015110/1. E.M.G. acknowledges support from the Royal Society of Edinburgh and Scottish Government and EPSRC Grant No. EP/T007214/1. Computations were performed using QuantumOptics.j1 [61].
[1] R. Blankenship, Molecular Mechanisms of Photosynthesis (Wiley, Hoboken, NJ, 2008).

[2] H. van Amerongen, L. Valkunas, and R. van Grondelle, Photosynthetic Excitons (World Scientific, Singapore, 2000).

[3] E. Pop, Energy dissipation and transport in nanoscale devices, Nano Res. 3, 147 (2010).

[4] J. Nelson, Physics of Solar Cells (Wiley, Hoboken, NJ, 2007).

[5] E. Romero, V. Novoderezhkin, and R. Grondelle, Quantum design of photosynthesis for bio-inspired solar-energy conversion, Nature (London) 543, 355 (2017).

[6] J.-L. Brédas, E. H. Sargent, and G. D. Scholes, Photovoltaic concepts inspired by coherence effects in photosynthetic systems, Nat. Mater. 16, 35 (2016).

[7] W. M. Brown and E. M. Gauger, Light harvesting with guideslide superabsorbing condensed-matter nanostructures, J. Phys. Chem. Lett. 10, 4323 (2019).

[8] L. S. Petrosyan, A. S. Kirakosyan, and T. V. Shahbazyan, Extraordinary Electron Transmission through a Periodic Array of Quantum Dots, Phys. Rev. Lett. 107, 196802 (2011).

[9] H.-P. Breuer and F. Petruccione, The Theory of Open Quantum Systems (Oxford University Press, Oxford, UK, 2002).

[10] M. Mohseni, P. Rebentrost, S. Lloyd, and A. Aspuru-Guzik, Environment-assisted quantum walks in photosynthetic energy transfer, J. Chem. Phys. 129, 174106 (2008).

[11] M. Walschaers, F. Schlawin, T. Wellens, and A. Buchleitner, Quantum transport on disordered and noisy networks: An interplay of structural complexity and uncertainty, Annu. Rev. Condens. Matter Phys. 7, 223 (2016).

[12] K. Head-Marsden and D. A. Mazziotti, Ensemble of Lindblad's trajectories for non-Markovian dynamics, Phys. Rev. A 99, 022109 (2019).

[13] M. B. Plenio and S. F. Huelga, Dephasing-assisted transport: Quantum networks and biomolecules, New J. Phys. 10, 113019 (2008).

[14] Y. Li, F. Caruso, E. Gauger, and S. C. Benjamin, 'Momentum rejuvenation' underlies the phenomenon of noise-assisted quantum energy flow, New J. Phys. 17, 013057 (2015).

[15] E. Zerah-Harush and Y. Dubi, Universal origin for environmentassisted quantum transport in exciton transfer networks, J. Phys. Chem. Lett. 9, 1689 (2018).

[16] P. Schijven, J. Kohlberger, A. Blumen, and O. Mülken, Modeling the quantum to classical crossover in topologically disordered networks, J. Phys. A: Math. Theor. 45, 215003 (2012).

[17] F. Caruso, Universally optimal noisy quantum walks on complex networks, New J. Phys. 16, 055015 (2014).

[18] J. M. Moix, M. Khasin, and J. Cao, Coherent quantum transport in disordered systems: I. The influence of dephasing on the transport properties and absorption spectra on one-dimensional systems, New J. Phys. 15, 085010 (2013).

[19] S. Mostarda, F. Levi, D. Prada-Gracia, F. Mintert, and F. Rao, Structure-dynamics relationship in coherent transport through disordered systems, Nat. Commun. 4, 2296 (2013).

[20] T. Scholak, T. Wellens, and A. Buchleitner, Optimal networks for excitonic energy transport, J. Phys. B: At., Mol. Opt. Phys. 44, 184012 (2011).

[21] T. Scholak, F. de Melo, T. Wellens, F. Mintert, and A. Buchleitner, Efficient and coherent excitation transfer across disordered molecular networks, Phys. Rev. E 83, 021912 (2011).

[22] T. Zech, R. Mulet, T. Wellens, and A. Buchleitner, Centrosymmetry enhances quantum transport in disordered molecular networks, New J. Phys. 16, 055002 (2014).

[23] G. C. Knee, P. Rowe, L. D. Smith, A. Troisi, and A. Datta, Structure-dynamics relation in physically-plausible multichromophore systems, J. Phys. Chem. Lett. 8, 2328 (2017).

[24] K. Claridge, D. Padula, and A. Troisi, On the arrangement of chromophores in light harvesting complexes: Chance versus design, Faraday Discuss. 221, 133 (2020).

[25] J. Cao and R. J. Silbey, Optimization of exciton trapping in energy transfer processes, J. Phys. Chem. A 113, 13825 (2009).

[26] G. Styliaris, N. Anand, L. Campos Venuti, and P. Zanardi, Quantum coherence and the localization transition, Phys. Rev. B 100, 224204 (2019).

[27] M. Mohseni, A. Shabani, S. Lloyd, Y. Omar, and H. Rabitz, Geometrical effects on energy transfer in disordered open quantum systems, J. Chem. Phys. 138, 204309 (2013).

[28] A. W. Chin, J. Prior, R. Rosenbach, F. Caycedo-Soler, S. F. Huelga, and M. B. Plenio, The role of non-equilibrium vibrational structures in electronic coherence and recoherence in pigment-protein complexes, Nat. Phys. 9, 113 (2013).

[29] E. K. Irish, R. Gómez-Bombarelli, and B. W. Lovett, Vibrationassisted resonance in photosynthetic excitation-energy transfer, Phys. Rev. A 90, 012510 (2014). 
[30] J. J. J. Roden, D. I. G. Bennett, and K. B. Whaley, Long-range energy transport in photosystem II, J. Chem. Phys. 144, 245101 (2016).

[31] J. K. Sowa, J. A. Mol, G. A. D. Briggs, and E. M. Gauger, Environment-assisted quantum transport through single-molecule junctions, Phys. Chem. Chem. Phys. 19, 29534 (2017).

[32] G. Candiotto, A. Torres, K. T. Mazon, and L. G. C. Rego, Charge generation in organic solar cells: Interplay of quantum dynamics, decoherence, and recombination, J. Phys. Chem. C 121, 23276 (2017).

[33] S. E. Venegas-Andraca, Quantum walks: A comprehensive review, Quantum Inf. Process. 11, 1015 (2012).

[34] M. K. Transtrum, B. B. Machta, K. S. Brown, B. C. Daniels, C. R. Myers, and J. P. Sethna, Perspective: Sloppiness and emergent theories in physics, biology, and beyond, J. Chem. Phys. 143, 010901 (2015).

[35] See Supplemental Material at http://link.aps.org/supplemental/ 10.1103/PhysRevResearch.3.L032001 for further details of our procedure and results as well as a discussion of related works and relevant experimental platforms.

[36] E. M. Gauger and J. Wabnig, Heat pumping with optically driven excitons, Phys. Rev. B 82, 073301 (2010).

[37] I. de Vega and D. Alonso, Dynamics of non-Markovian open quantum systems, Rev. Mod. Phys. 89, 015001 (2017).

[38] F. A. Pollock, D. P. S. McCutcheon, B. W. Lovett, E. M. Gauger, and A. Nazir, A multi-site variational master equation approach to dissipative energy transfer, New J. Phys. 15, 075018 (2013).

[39] A. Kolli, A. Nazir, and A. Olaya-Castro, Electronic excitation dynamics in multichromophoric systems described via a polaron-representation master equation, J. Chem. Phys. 135, 154112 (2011).

[40] S. Jang, Theory of multichromophoric coherent resonance energy transfer: A polaronic quantum master equation approach, J. Chem. Phys. 135, 034105 (2011).

[41] Y. Tanimura and R. Kubo, Time evolution of a quantum system in contact with a nearly Gaussian-Markoffian noise bath, J. Phys. Soc. Jpn. 58, 101 (1989).

[42] J. Zhu, S. Kais, P. Rebentrost, and A. Aspuru-Guzik, Modified scaled hierarchical equation of motion approach for the study of quantum coherence in photosynthetic complexes, J. Phys. Chem. B 115, 1531 (2011).

[43] J. Prior, A. W. Chin, S. F. Huelga, and M. B. Plenio, Efficient Simulation of Strong System-Environment Interactions, Phys. Rev. Lett. 105, 050404 (2010).

[44] A. D. Somoza, O. Marty, J. Lim, S. F. Huelga, and M. B. Plenio, Dissipation-Assisted Matrix Product Factorization, Phys. Rev. Lett. 123, 100502 (2019).

[45] A. Strathearn, B. W. Lovett, and P. Kirton, Efficient real-time path integrals for non-Markovian spin-boson models, New J. Phys. 19, 093009 (2017).
[46] A. Strathearn, P. Kirton, D. Kilda, J. Keeling, and B. W. Lovett, Efficient non-Markovian quantum dynamics using timeevolving matrix product operators, Nat. Commun. 9, 3322 (2018).

[47] M. R. Jørgensen and F. A. Pollock, Exploiting the Causal Tensor Network Structure of Quantum Processes to Efficiently Simulate Non-Markovian Path Integrals, Phys. Rev. Lett. 123, 240602 (2019).

[48] S. Amari and H. Nagaoka, Methods of Information Geometry, Translations of Mathematical Monographs (American Mathematical Society, Providence, RI, 2007).

[49] E. Dufresne, H. A. Harrington, and D. V. Raman, The geometry of sloppiness, J. Algebr. Stat. 9, 30 (2018).

[50] B. B. Machta, R. Chachra, M. K. Transtrum, and J. P. Sethna, Parameter space compression underlies emergent theories and predictive models, Science 342, 604 (2013).

[51] J. J. Waterfall, F. P. Casey, R. N. Gutenkunst, K. S. Brown, C. R. Myers, P. W. Brouwer, V. Elser, and J. P. Sethna, Sloppy-Model Universality Class and the Vandermonde Matrix, Phys. Rev. Lett. 97, 150601 (2006).

[52] K. S. Brown and J. P. Sethna, Statistical mechanical approaches to models with many poorly known parameters, Phys. Rev. E 68, 021904 (2003).

[53] M. K. Transtrum, B. B. Machta, and J. P. Sethna, Geometry of nonlinear least squares with applications to sloppy models and optimization, Phys. Rev. E 83, 036701 (2011).

[54] S. Tomasi and I. Kassal, Classification of coherent enhancements of light-harvesting processes, J. Phys. Chem. Lett. 11, 2348 (2020).

[55] C. Emary and R. Aguado, Quantum versus classical counting in non-Markovian master equations, Phys. Rev. B 84, 085425 (2011).

[56] S. Krastanov, K. Head-Marsden, S. Zhou, S. T. Flammia, L. Jiang, and P. Narang, Unboxing quantum black box models: Learning non-Markovian dynamics, arXiv:2009.03902.

[57] C. Guo, K. Modi, and D. Poletti, Tensor-network-based machine learning of non-Markovian quantum processes, Phys. Rev. A 102, 062414 (2020).

[58] I. A. Luchnikov, S. V. Vintskevich, D. A. Grigoriev, and S. N. Filippov, Machine Learning Non-Markovian Quantum Dynamics, Phys. Rev. Lett. 124, 140502 (2020).

[59] M. K. Transtrum and P. Qiu, Model Reduction by Manifold Boundaries, Phys. Rev. Lett. 113, 098701 (2014).

[60] S. Davidson, A. Fruchtman, F. A. Pollock, and E. M. Gauger, The dark side of energy transport along excitonic wires: Onsite energy barriers facilitate efficient, vibrationally mediated transport through optically dark subspaces, J. Chem. Phys. 153, 134701 (2020).

[61] S. Krämer, D. Plankensteiner, L. Ostermann, and H. Ritsch, QuantumOptics.jl: A Julia framework for simulating open quantum systems, Comput. Phys. Commun. 227, 109 (2018). 